PROCEEDINGS OF THE

AMERICAN MATHEMATICAL SOCIETY

Volume 131, Number 1, Pages 309-318

S 0002-9939(02)06501-2

Article electronically published on May 13, 2002

\title{
GAMES AND GENERAL DISTRIBUTIVE LAWS IN BOOLEAN ALGEBRAS
}

\author{
NATASHA DOBRINEN
}

(Communicated by Carl G. Jockusch, Jr.)

\begin{abstract}
The games $\mathcal{G}_{1}^{\eta}(\kappa)$ and $\mathcal{G}_{<\lambda}^{\eta}(\kappa)$ are played by two players in $\eta^{+}$complete and $\max \left(\eta^{+}, \lambda\right)$-complete Boolean algebras, respectively. For cardinals $\eta, \kappa$ such that $\kappa^{<\eta}=\eta$ or $\kappa^{<\eta}=\kappa$, the $(\eta, \kappa)$-distributive law holds in a Boolean algebra $\mathbf{B}$ iff Player 1 does not have a winning strategy in $\mathcal{G}_{1}^{\eta}(\kappa)$. Furthermore, for all cardinals $\kappa$, the $(\eta, \infty)$-distributive law holds in $\mathbf{B}$ iff Player 1 does not have a winning strategy in $\mathcal{G}_{1}^{\eta}(\infty)$. More generally, for cardinals $\eta, \lambda, \kappa$ such that $\left(\kappa^{<\lambda}\right)^{<\eta}=\eta$, the $(\eta,<\lambda, \kappa)$-distributive law holds in $\mathbf{B}$ iff Player 1 does not have a winning strategy in $\mathcal{G}_{<\lambda}^{\eta}(\kappa)$. For $\eta$ regular and $\lambda \leq \min (\eta, \kappa), \diamond_{\eta^{+}}$implies the existence of a Suslin algebra in which $\mathcal{G}_{<\lambda}^{\eta}(\kappa)$ is undetermined.
\end{abstract}

\section{INTRODUCTION}

Games between two players arise naturally in the study of distributive laws in Boolean algebras. Jech [3] obtained a game-theoretic characterization of the $(\omega, \infty)$ distributive law using the game $\mathcal{G}$ which is played by two players in a complete Boolean algebra $\mathbf{B}$ as follows: Player 1 begins the game by choosing some $a_{0} \in \mathbf{B}^{+}$ (where $\mathbf{B}^{+}=\mathbf{B} \backslash\{\mathbf{0}\}$ ); then Player 2 chooses some $b_{0} \in \mathbf{B}^{+}$such that $b_{0} \leq a_{0}$. The two players take turns choosing elements $a_{n}, b_{n} \in \mathbf{B}^{+}$to form a descending sequence

$$
a_{0} \geq b_{0} \geq a_{1} \geq b_{1} \geq \cdots \geq a_{n} \geq b_{n} \geq \cdots .
$$

Player 1 wins the play (1) iff the sequence (1) has no lower bound in $\mathbf{B}^{+}$. Jech showed that the $(\omega, \infty)$-distributive law holds in $\mathbf{B}$ iff Player 1 does not have a winning strategy in $\mathcal{G}$. A few years later, Foreman [1] obtained a game-theoretic characterization of the $(\eta, \infty)$-distributive law for successor cardinals $\eta$. For a partial ordering $\mathbf{P}$, Fuchino, Mildenberger, Shelah, and Vojtáš 2] defined the game $G(\mathbf{P}, \eta)$, which is played just like $\mathcal{G}$, except that it is played in $\eta$-many rounds, where Player 1 plays first at each limit ordinal. They showed that if $\mathbf{P}$ is separative, then for all cardinals $\eta$ the $(\eta, \infty)$-distributive law holds in r.o.(P) (the regular open algebra of $\mathbf{P}$ ) iff Player 1 does not have a winning strategy in $G(\mathbf{P}, \eta)$.

Interested in the second parameter of the $(\omega, \kappa)$-distributive law, Jech [4] defined the game $\mathcal{G}_{1}(\kappa)$ to be played in a complete Boolean algebra. At the beginning of the game, Player 1 chooses some $a \in \mathbf{B}^{+}$which is then fixed throughout the $\omega$-many

Received by the editors November 17, 2000 and, in revised form, August 23, 2001.

2000 Mathematics Subject Classification. Primary 03G05, 06E25; Secondary 03E40.

Key words and phrases. Boolean algebra, distributivity, games.

(C)2002 American Mathematical Society 
rounds. On the $n$-th round, Player 1 chooses $W_{n} \subseteq \mathbf{B}^{+}$, a partition of $a$ such that $\left|W_{n}\right| \leq \kappa$; then Player 2 chooses some $b_{n} \in W_{n}$. Player 1 wins the play

$$
\left\langle a, W_{0}, b_{0}, W_{1}, b_{1}, \ldots, W_{n}, b_{n}, \ldots\right\rangle
$$

iff $\bigwedge_{n<\omega} b_{n}=\mathbf{0}$. Jech showed that, for all cardinals $\kappa$, the $(\omega, \kappa)$-distributive law holds in B iff Player 1 does not have a winning strategy in $\mathcal{G}_{1}(\kappa)$.

In $\S 1$, we present the game $\mathcal{G}_{1}^{\eta}(\kappa)$ which generalizes $\mathcal{G}_{1}(\kappa)$ to plays of uncountable length. We show that, for cardinals $\eta, \kappa$ such that $\kappa^{<\eta}=\eta$ or $\kappa^{<\eta}=\kappa$, the $(\eta, \kappa)$-distributive law is characterized by the non-existence of a winning strategy for Player 1 in $\mathcal{G}_{1}^{\eta}(\kappa)$. It immediately follows that the $(\eta, \infty)$-distributive law holds in $\mathbf{B}$ iff Player 1 does not have a winning strategy in $\mathcal{G}_{1}^{\eta}(\infty)$. This yields a characterization of $(\eta, \infty)$-distributivity different than the characterization obtained by Fuchino, Mildenberger, Shelah and Vojtáš in [2]. Moreover, it shows the equivalence of the existence of a winning strategy for Player 1 in the games $G\left(\mathbf{B}^{+}, \eta\right)$ and $\mathcal{G}_{1}^{\eta}(\infty)$.

In $\S 2$, we present the game $\mathcal{G}_{<\lambda}^{\eta}(\kappa)$, played similarly to $\mathcal{G}_{1}^{\eta}(\kappa)$, except that now Player 2 chooses less than $\lambda$-many pieces from each of Player 1's partitions. We show that, for cardinals $\eta, \kappa, \lambda$ such that $\left(\kappa^{<\lambda}\right)^{<\eta}=\eta$, the $(\eta,<\lambda, \kappa)$-distributive law holds iff Player 1 does not have a winning strategy in $\mathcal{G}_{<\lambda}^{\eta}(\kappa)$. Since the $(\eta,<\omega, \kappa)$ distributive law is the same as the weak $(\eta, \kappa)$-distributive law, this yields a gametheoretic characterization of the weak $(\eta, \kappa)$-distributive law for cardinals $\eta, \kappa$ such that $\kappa^{<\eta}=\eta$. Moreover, for regular infinite cardinals $\eta$ and cardinals $\lambda, \kappa$ with $\lambda \leq \min (\eta, \kappa)$, we use $\nabla_{\eta^{+}}$to construct an $\eta^{+}$-Suslin algebra in which $\mathcal{G}_{1}^{\eta}(\kappa)$ and $\mathcal{G}_{<\lambda}^{\eta}(\kappa)$ are undetermined.

\section{The $(\eta, \kappa)$-Distributive LaW And the Game $\mathcal{G}_{1}^{\eta}(\kappa)$}

We begin by reviewing the following basic distributive law. Throughout this paper, let $\mathbf{B}$ denote a Boolean algebra.

Definition $1.1(7])$. B satisfies the $(\eta, \kappa)$-distributive law $((\eta, \kappa)$-d.l. $)$ if for each $|I| \leq \eta,|J| \leq \kappa$, and family $\left(b_{i j}\right)_{i \in I, j \in J}$ of elements of $\mathbf{B}$,

$$
\bigwedge_{i \in I} \bigvee_{j \in J} b_{i j}=\bigvee_{f: I \rightarrow J} \bigwedge_{i \in I} b_{i f(i)}
$$

provided that $\bigvee_{j \in J} b_{i j}$ for each $i \in I, \bigwedge_{i \in I} \bigvee_{j \in J} b_{i j}$, and $\bigwedge_{i \in I} b_{i f(i)}$ for each $f$ : $I \rightarrow J$ exist in $\mathbf{B}$. We say that $\mathbf{B}$ is $(\eta, \infty)$-distributive if it satisfies the $(\eta, \kappa)$-d.l. for all cardinals $\kappa$.

Jech showed that the $(\eta, \kappa)$-d.l. is equivalent to the following forcing property.

Proposition 1.1 (5]). If $\mathbf{B}$ is complete, then $\mathbf{B}$ is $(\eta, \kappa)$-distributive if and only if every function from $\eta$ to $\kappa$ in the generic extension $V[G]$ belongs to the ground model $V$. B is $(\eta, \infty)$-distributive if and only if every function $f: \eta \rightarrow V$ in $V[G]$ is in $V$.

The following generalizes Jech's game $\mathcal{G}_{1}(\kappa)$ to plays of uncountable length.

Definition 1.2. Let $\kappa$ be any cardinal and $\eta$ be any infinite cardinal. The game $\mathcal{G}_{1}^{\eta}(\kappa)$ is played between two players in an $\eta^{+}$-complete Boolean algebra $\mathbf{B}$ as follows: At the beginning of the game, Player 1 (P1) chooses some $a \in \mathbf{B}^{+}$which is fixed throughout the $\eta$-many rounds. For $\alpha<\eta$, the $\alpha$-th round is played as follows: P1 
chooses a partition $W_{\alpha}$ of $a$ such that $\left|W_{\alpha}\right| \leq \kappa$; then Player 2 (P2) chooses some $b_{\alpha} \in W_{\alpha}$. In this manner, the two players construct a sequence of length $\eta$

$$
\left\langle a, W_{0}, b_{0}, W_{1}, b_{1}, \ldots, W_{\alpha}, b_{\alpha}, \ldots: \alpha<\eta\right\rangle
$$

called a play of the game. P1 wins the play (4) if and only if

$$
\bigwedge_{\alpha<\eta} b_{\alpha}=\mathbf{0} .
$$

A strategy for P1 is a function $\sigma:\{\mathbf{0}\} \cup\left(\mathbf{B}^{+}\right)^{<\eta} \rightarrow\left[\mathbf{B}^{+}\right]^{\leq \kappa}$ such that $\sigma(\mathbf{0})=\{a\}$ and for each $\left\langle b_{\alpha}: \alpha<\beta\right\rangle \in\left(\mathbf{B}^{+}\right)^{<\eta}, \sigma\left(\left\langle b_{\alpha}: \alpha<\beta\right\rangle\right)$ is a partition of $a$. $\sigma$ is a winning strategy if $\mathrm{P} 1$ wins every time $\mathrm{P} 1$ follows $\sigma$.

A strategy for P2 is a function $\tau:\left(\left[\mathbf{B}^{+}\right]^{\leq \kappa}\right)^{<\eta} \rightarrow \mathbf{B}^{+}$such that for each $\left\langle W_{\alpha}\right.$ : $\alpha \leq \beta\rangle \in\left(\left[\mathbf{B}^{+}\right] \leq \kappa\right)<\eta, \tau\left(\left\langle W_{\alpha}: \alpha \leq \beta\right\rangle\right) \in W_{\beta} . \tau$ is a winning strategy if whenever P2 plays by $\tau, \mathrm{P} 2$ wins.

$\mathcal{G}_{1}^{\eta}=\mathcal{G}_{1}^{\eta}(\infty)$ is the game played as above, except now $\mathrm{P} 1$ can choose partitions of any size. We note that Jech's game $\mathcal{G}_{1}(\kappa)$ is the same as our game $\mathcal{G}_{1}^{\omega}(\kappa)$.

Remark. $\mathcal{G}_{1}^{\eta}$ can be played in a partial ordering $\mathbf{P}$ in the natural way: At the beginning of the game, $\mathrm{P} 1$ chooses some fixed $p \in \mathbf{P}$. On the $\alpha$-th round, $\mathrm{P} 1$ chooses a maximal incompatible subset $M_{\alpha} \subseteq \mathbf{P}$ below $p$; then $\mathrm{P} 2$ chooses one element $p_{\alpha} \in M_{\alpha}$. This constructs the sequence

$$
\left\langle p, M_{0}, p_{0}, M_{1}, p_{1}, \ldots, M_{\alpha}, p_{\alpha}, \ldots: \alpha<\eta\right\rangle .
$$

P1 wins the play (6) if and only if $\forall q \in \mathbf{P}, \exists \alpha<\eta$ such that $q \not \leq p_{\alpha}$. If $\mathbf{P}$ is separative, then the existence of a winning strategy for P1 (P2) is invariant when passing to r.o. $(\mathbf{P})$.

One can easily show that if P2 has a winning strategy in $\mathcal{G}_{1}^{\eta}(\kappa)\left(\mathcal{G}_{1}^{\eta}\right)$, then the $(\eta, \kappa)$-d.l. $((\eta, \infty)$-d.l. $)$ holds. Jech obtained the following characterization of the $(\omega, \kappa)$-d.l.

Theorem 1.2 ([4]). If $\mathbf{B}$ is complete, then the $(\omega, \kappa)$-d.l. holds in $\mathbf{B}$ if and only if $P 1$ does not have a winning strategy in $\mathcal{G}_{1}^{\omega}(\kappa)$.

The "if" direction easily generalizes to all pairs of cardinals $\eta, \kappa$.

Theorem 1.3. If $\mathbf{B}$ is $\eta^{+}$-complete and the $(\eta, \kappa)$-d.l. fails in $\mathbf{B}$, then P1 has a winning strategy in $\mathcal{G}_{1}^{\eta}(\kappa)$.

Proof. Follows from Theorem 2.2.

We have obtained the following partial converse to Theorem 1.3.

Theorem 1.4. If $\mathbf{B}$ is $\eta^{+}$-complete and $P 1$ has a winning strategy in $\mathcal{G}_{1}^{\eta}(\kappa)$, then

(1) the $\left(\kappa^{<\eta}, \kappa\right)$-d.l. fails;

(2) the $\left(\eta, \kappa^{<\eta}\right)$-d.l. fails.

Proof. (1) follows from Theorem 2.3. The proof of (2) uses ideas from Jech's proof of Theorem 1.2, the main difference being that here, limit ordinals must be treated with care, as in Case 1 , below. Let $\sigma$ be a winning strategy for $\mathrm{P} 1$ in $\mathcal{G}_{1}^{\eta}(\kappa)$. Let $\{a\}=\sigma(\mathbf{0})$ and $P_{0}=\sigma(\langle\rangle)$. For each $x_{0} \in P_{0}$, let $W_{1}\left(\left\langle x_{0}\right\rangle\right)=\left\{x_{0} \wedge z: z \in \sigma\left(\left\langle x_{0}\right\rangle\right)\right\}$. Let $P_{1}=\bigcup\left\{W_{1}\left(\left\langle x_{0}\right\rangle\right): x_{0} \in \sigma(\langle\rangle)\right\}$. For each $x_{1} \in \sigma\left(\left\langle x_{0}\right\rangle\right)$, let $W_{2}\left(\left\langle x_{0}, x_{1}\right\rangle\right)=$ $\left\{x_{0} \wedge x_{1} \wedge z: z \in \sigma\left(\left\langle x_{0}, x_{1}\right\rangle\right)\right\}$. Let $P_{2}=\bigcup\left\{W_{2}\left(\left\langle x_{0}, x_{1}\right\rangle\right): x_{0} \in \sigma(\langle\rangle), x_{1} \in \sigma\left(\left\langle x_{0}\right\rangle\right)\right\}$. 
In general, given $\alpha<\eta$ and a sequence $\left\langle x_{\beta}: \beta<\alpha\right\rangle \in\left(\mathbf{B}^{+}\right)^{\alpha}$ such that $\forall \beta<\alpha$ $x_{\beta} \in \sigma\left(\left\langle x_{\gamma}: \gamma<\beta\right\rangle\right)$, let

$$
W_{\alpha}\left(\left\langle x_{\beta}: \beta<\alpha\right\rangle\right)=\left\{\left(\bigwedge_{\beta<\alpha} x_{\beta}\right) \wedge z: z \in \sigma\left(\left\langle x_{\beta}: \beta<\alpha\right\rangle\right)\right\},
$$

and let

$$
P_{\alpha}=\bigcup\left\{W_{\alpha}\left(\left\langle x_{\beta}: \beta<\alpha\right\rangle\right): \forall \beta<\alpha, x_{\beta} \in \sigma\left(\left\langle x_{\gamma}: \gamma<\beta\right\rangle\right)\right\} .
$$

Note that

(a) $\forall \alpha<\eta, P_{\alpha} \subseteq \mathbf{B}^{+}$is pairwise disjoint and $\left|P_{\alpha}\right| \leq \kappa^{\alpha+1} \leq \kappa^{<\eta}$.

(b) If $\beta<\alpha<\eta$, then $\forall x \in P_{\alpha} \exists y \in P_{\beta}$ such that $y \geq x$.

(c) If $\alpha<\eta$ and $\forall \beta<\alpha, x_{\beta} \in \sigma\left(\left\langle x_{\gamma}: \gamma<\beta\right\rangle\right)$, then $\bigvee W_{\alpha}\left(\left\langle x_{\beta}: \beta<\alpha\right\rangle\right)=$ $\bigwedge_{\beta<\alpha} x_{\beta}$.

(d) $\forall \alpha<\eta, \bigvee P_{\alpha+1}=\bigvee P_{\alpha}$

(e) If (i) $\beta \leq \gamma<\eta$;

(ii) $\left\langle x_{\zeta}: \zeta<\beta\right\rangle,\left\langle y_{\zeta}: \zeta<\gamma\right\rangle$ are sequences such that $\forall \zeta<\beta, x_{\zeta} \in \sigma\left(\left\langle x_{\theta}: \theta<\zeta\right\rangle\right)$ and $y_{\zeta} \in \sigma\left(\left\langle y_{\theta}: \theta<\zeta\right\rangle\right)$; and

(iii) $\exists \delta \leq \beta$ such that $x_{\delta} \neq y_{\delta}$;

then $\bigwedge_{\zeta<\beta} x_{\zeta} \wedge \bigwedge_{\zeta<\gamma} y_{\zeta}=\mathbf{0}$.

(a)-(d) are clear. For (e), if $\delta \leq \beta$ is least such that $x_{\delta} \neq y_{\delta}$, then $x_{\delta}, y_{\delta} \in$ $\sigma\left(\left\langle x_{\zeta}: \zeta<\delta\right\rangle\right)$, a partition of $a$, so $x_{\delta} \wedge y_{\delta}=\mathbf{0}$.

Claim. The $\left(\eta, \kappa^{<\eta}\right)$-d.l. fails for the collection $P_{\alpha},(\alpha<\eta)$. By (a), we can index the elements of $P_{\alpha}$ using the index set $\kappa^{<\eta}$ so that $P_{\alpha}=\left\{b_{\alpha, \gamma}: \gamma<\kappa^{<\eta}\right\}$, allowing repetitions.

Case 1. $\exists \alpha<\eta$ for which $\bigvee P_{\alpha}<a$. Let $\alpha$ be least such that $\bigvee P_{\alpha}<a$. (d) implies $\alpha$ is a limit ordinal. We will show that the $\left(\alpha, \kappa^{<\eta}\right)$-d.l. fails for the partitions $P_{\beta}(\beta<\alpha)$ of $a$. Since $\bigwedge_{\beta<\alpha} \bigvee P_{\beta}=a$, it suffices to show that

$$
\bigvee_{f: \alpha \rightarrow \kappa<\eta} \bigwedge_{\beta<\alpha} b_{\beta, f(\beta)} \leq \bigvee P_{\alpha}
$$

Let $f: \alpha \rightarrow \kappa^{<\eta}$ be given. $\forall \beta<\alpha$, there is a sequence $\left\langle x_{\zeta}^{\beta}: \zeta \leq \beta\right\rangle$ such that $b_{\beta, f(\beta)}=\bigwedge_{\zeta \leq \beta} x_{\zeta}^{\beta}$ and $\forall \zeta \leq \beta \quad x_{\zeta}^{\beta} \in \sigma\left(\left\langle x_{\gamma}^{\beta}: \gamma<\zeta\right\rangle\right)$.

Suppose $\forall \zeta \leq \beta<\gamma<\alpha, x_{\zeta}^{\beta}=x_{\zeta}^{\gamma} . \forall \beta<\alpha$, let $x_{\beta}$ denote $x_{\beta}^{\beta}$. Then $\forall \beta<\alpha$, $x_{\beta} \in \sigma\left(\left\langle x_{\zeta}: \zeta<\beta\right\rangle\right)$, so (c) implies $\bigwedge_{\beta<\alpha} x_{\beta}=\bigvee W_{\alpha}\left(\left\langle x_{\beta}: \beta<\alpha\right\rangle\right)$. Therefore,

$$
\bigwedge_{\beta<\alpha} b_{\beta, f(\beta)}=\bigwedge_{\beta<\alpha} \bigwedge_{\zeta \leq \beta} x_{\zeta}^{\beta}=\bigwedge_{\beta<\alpha} x_{\beta}=\bigvee W_{\alpha}\left(\left\langle x_{\beta}: \beta<\alpha\right\rangle\right) \leq \bigvee P_{\alpha}
$$

Otherwise, $\exists \delta \leq \beta<\gamma<\alpha$ such that $x_{\delta}^{\beta} \neq x_{\delta}^{\gamma}$. In this case, (e) implies

$$
\bigwedge_{\zeta<\alpha} b_{\zeta, f(\zeta)} \leq b_{\beta, f(\beta)} \wedge b_{\gamma, f(\gamma)}=\bigwedge_{\zeta \leq \beta} x_{\zeta}^{\beta} \wedge \bigwedge_{\zeta \leq \gamma} x_{\zeta}^{\gamma}=\mathbf{0} .
$$

Since (10) and (11) hold for all $f: \alpha \rightarrow \kappa^{<\eta},(9)$ holds. Therefore, the $\left(\alpha, \kappa^{<\eta}\right)$ d.l. fails.

Case 2. For each $\alpha<\eta, \bigvee P_{\alpha}=a$. (b) implies $\forall \beta<\alpha<\eta, P_{\alpha}$ is a refinement of $P_{\beta}$. Let $f: \eta \rightarrow \kappa^{<\eta}$ be given. We will show that $\bigwedge_{\alpha<\eta} b_{\alpha, f(\alpha)}=\mathbf{0}$. If $\exists \alpha<\eta$ such that $b_{\alpha, f(\alpha)}=\mathbf{0}$, then we are done; so assume $\forall \alpha<\eta, b_{\alpha, f(\alpha)} \neq \mathbf{0}$. 
Suppose $\exists \beta<\alpha<\eta$ such that $b_{\beta, f(\beta)} \nsupseteq b_{\alpha, f(\alpha)}$. Then, $b_{\beta, f(\beta)} \wedge b_{\alpha, f(\alpha)}=\mathbf{0}$, since $P_{\alpha}$ is a refinement of $P_{\beta}$. Hence, $\bigwedge_{\alpha<\eta} b_{\alpha, f(\alpha)}=\mathbf{0}$.

Otherwise, $\forall \beta<\alpha<\eta, b_{\beta, f(\beta)} \geq b_{\alpha, f(\alpha)}>\mathbf{0}$. Again, $\forall \beta<\alpha$ let $\left\langle x_{\zeta}^{\beta}: \zeta<\beta\right\rangle$ be the sequence such that $b_{\beta, f(\beta)}=\bigwedge_{\zeta \leq \beta} x_{\zeta}^{\beta}$ and $\forall \zeta \leq \beta x_{\zeta}^{\beta} \in \sigma\left(\left\langle x_{\gamma}^{\beta}: \gamma<\zeta\right\rangle\right)$. We claim that

$$
\forall \zeta \leq \beta<\alpha<\eta, \quad x_{\zeta}^{\beta}=x_{\zeta}^{\alpha} .
$$

If (12) fails, then $\exists \delta, \beta, \alpha$ with $\delta \leq \beta<\alpha<\eta$ such that $x_{\delta}^{\beta} \neq x_{\delta}^{\alpha}$. Then $b_{\beta, f(\beta)} \wedge b_{\alpha, f(\alpha)}=\mathbf{0}$, by (e). But this implies $b_{\alpha, f(\alpha)}=\mathbf{0}$, since $b_{\alpha, f(\alpha)} \leq b_{\beta, f(\beta)}$. Contradiction. Thus, (12) holds. $\forall \alpha<\eta$ let $x_{\alpha}$ denote $x_{\alpha}^{\alpha}$. Then

$$
\left\langle a, \sigma(\langle\rangle), x_{0}, \sigma\left(\left\langle x_{0}\right\rangle\right), x_{1}, \ldots, \sigma\left(\left\langle x_{\beta}: \beta<\alpha\right\rangle\right), x_{\alpha}, \ldots: \alpha<\eta\right\rangle
$$

is a play in $\mathcal{G}_{1}^{\eta}(\kappa)$ in which $\mathrm{P} 1$ follows $\sigma$. So,

$$
\bigwedge_{\alpha<\eta} b_{\alpha, f(\alpha)}=\bigwedge_{\alpha<\eta} \bigwedge_{\zeta \leq \alpha} x_{\zeta}^{\alpha}=\bigwedge_{\alpha<\eta} x_{\alpha}=\mathbf{0}
$$

Since $f: \eta \rightarrow \kappa^{<\eta}$ was arbitrary,

$$
\bigvee_{f: \eta \rightarrow \kappa<\eta} \bigwedge_{\alpha<\eta} b_{\alpha, f(\alpha)}=\mathbf{0}<a .
$$

Thus, the $\left(\eta, \kappa^{<\eta}\right)$-d.l. fails for the partitions $P_{\alpha}(\alpha<\eta)$ of $a$.

Whether the full converse of Theorem 1.4 holds is unknown. However, for certain pairs of cardinals $\eta, \kappa$ we have the following:

Corollary 1.5. If $\mathbf{B}$ is $\eta^{+}$-complete and $\kappa^{<\eta}=\eta$ or $\kappa^{<\eta}=\kappa$, then the $(\eta, \kappa)$-d.l. holds in $\mathbf{B}$ if and only if P1 does not have a winning strategy in $\mathcal{G}_{1}^{\eta}(\kappa)$.

From Corollary 1.5, we obtain a characterization of the $(\eta, \infty)$-d.l. which differs from the one given in [2].

Corollary 1.6. If $\mathbf{B}$ is $\eta^{+}$-complete, then the $(\eta, \infty)$-d.l. holds if and only if P1 does not have a winning strategy in $\mathcal{G}_{1}^{\eta}$.

Corollary 1.6 implies that the existence of a winning strategy for P1 in $\mathcal{G}_{1}^{\eta}$ is equivalent to the existence of a winning strategy for $\mathrm{P} 1$ in the game $G\left(\mathbf{B}^{+}, \eta\right)$ in [2].

Assuming GCH, Corollary 1.5 gives a characterization of the $(\eta, \kappa)$-d.l. for all $\kappa<\eta$ and for certain $\kappa \geq \eta$.

Corollary $1.7(\mathrm{GCH})$. If $\mathbf{B}$ is $\eta^{+}$-complete, then $\forall \kappa<\eta$ and $\forall \kappa$ with $\operatorname{cf}(\kappa) \geq \eta$, the $(\eta, \kappa)$-d.l. holds if and only if P1 does not have a winning strategy in $\mathcal{G}_{1}^{\eta}(\kappa)$.

Example 1. If $\eta$ is regular and $\nabla_{\eta^{+}}$holds, then there is an $\eta^{+}$-Suslin algebra in which neither player has a winning strategy in $\mathcal{G}_{1}^{\eta}(\kappa)$. (This follows from Example 2 with $\lambda=2$.)

Remark. $\eta$ regular and $\diamond_{\eta^{+}}$imply that the existence of a winning strategy for P2 in $\mathcal{G}_{1}^{\eta}(\kappa)$ is strictly stronger than the $(\eta, \kappa)$-d.l. Example 2 in $\S 2$ will shed more light on the difference between their relative strengths. 


\section{The $(\eta,<\lambda, \kappa)$-Distributive LAW AND The Game $\mathcal{G}_{<\lambda}^{\eta}(\kappa)$}

Three-parameter distributivity is the natural generalization of weak distributivity and is defined as follows.

Definition 2.1 ([5]). B satisfies the $(\eta,<\lambda, \kappa)$-distributive law $((\eta,<\lambda, \kappa)$-d.l.) if for each $|I| \leq \eta,|J| \leq \kappa$, and family $\left(b_{i j}\right)_{i \in I, j \in J}$ of elements of $\mathbf{B}$,

$$
\bigwedge_{i \in I} \bigvee_{j \in J} b_{i j}=\bigvee_{f: I \rightarrow[J]<\lambda} \bigwedge_{i \in I} \bigvee_{j \in f(i)} b_{i j},
$$

provided that $\bigvee_{j \in J} b_{i j}$ for each $i \in I, \bigwedge_{i \in I} \bigvee_{j \in J} b_{i j}$, and $\bigwedge_{i \in I} \bigvee_{j \in f(i)} b_{i j}$ for each $f: I \rightarrow[J]^{<\lambda}$ exist in $\mathbf{B}$. We say that $\mathbf{B}$ is $(\eta,<\lambda, \infty)$-distributive if it satisfies the $(\eta,<\lambda, \kappa)$-d.l. for all cardinals $\kappa$.

Note. The $(\eta, \kappa)$-d.l. is the same as the $(\eta,<2, \kappa)$-d.l., and the weak $(\eta, \kappa)$-d.l. is the same as the $(\eta,<\omega, \kappa)$-d.l.

$(\eta,<\lambda, \kappa)$-distributivity is equivalent to the following forcing property.

Proposition 2.1. If $\mathbf{B}$ is complete, then $\mathbf{B}$ is $(\eta,<\lambda, \kappa)$-distributive if and only if for every function $g: \eta \rightarrow \kappa$ in $V[G]$ there is a function $f: \eta \rightarrow[\kappa]^{<\lambda}$ in $V$ such that $\forall \alpha<\eta, g(\alpha) \in f(\alpha)$. B is $(\eta,<\lambda, \infty)$-distributive if and only if for every function $g: \eta \rightarrow V$ in $V[G]$ there is some function $f: \eta \rightarrow[V]^{<\lambda}$ such that $\forall \alpha<\eta$, $g(\alpha) \in f(\alpha)$.

The following game corresponds naturally to the $(\eta,<\lambda, \kappa)$-d.l.

Definition 2.2. Let $\eta, \kappa$ be infinite cardinals and $\lambda$ be a cardinal such that $2 \leq$ $\lambda \leq \kappa$. The game $\mathcal{G}_{<\lambda}^{\eta}(\kappa)$ is played between two players in a $\max \left(\eta^{+}, \lambda\right)$-complete Boolean algebra $\mathbf{B}$ as follows: At the beginning of the game, P1 chooses some $a \in \mathbf{B}^{+}$. For $\alpha<\eta$, the $\alpha$-th round is played as follows: P1 chooses a partition $W_{\alpha}$ of $a$ such that $\left|W_{\alpha}\right| \leq \kappa$; then P2 chooses some $F_{\alpha} \in\left[W_{\alpha}\right]^{<\lambda}$. In this manner, the two players construct a sequence of length $\eta$

$$
\left\langle a, W_{0}, F_{0}, W_{1}, F_{1}, \ldots, W_{\alpha}, F_{\alpha}, \ldots: \alpha<\eta\right\rangle,
$$

called a play of the game. P1 wins the play (17) if and only if

$$
\bigwedge_{\alpha<\eta} \bigvee F_{\alpha}=\mathbf{0}
$$

A strategy for $P 1$ is a function $\sigma:\{\mathbf{0}\} \cup\left(\left[\mathbf{B}^{+}\right]^{<\lambda}\right)^{<\eta} \rightarrow\left[\mathbf{B}^{+}\right]^{\leq \kappa}$ such that $\sigma(\mathbf{0})=\{a\}$ and for each $\left\langle F_{\alpha}: \alpha<\beta\right\rangle \in\left(\left[\mathbf{B}^{+}\right]^{<\lambda}\right)^{<\eta}, \sigma\left(\left\langle F_{\alpha}: \alpha<\beta\right\rangle\right)$ is a partition of $a . \sigma$ is a winning strategy if $\mathrm{P} 1$ wins every time $\mathrm{P} 1$ follows $\sigma$.

A strategy for P2 is a function $\tau:\left(\left[\mathbf{B}^{+}\right]^{\leq \kappa}\right)^{<\eta} \rightarrow\left[\mathbf{B}^{+}\right]^{<\lambda}$ such that for each $\left\langle W_{\alpha}: \alpha \leq \beta\right\rangle \in\left(\left[\mathbf{B}^{+}\right]^{\leq \kappa}\right)^{<\eta}, \tau\left(\left\langle W_{\alpha}: \alpha \leq \beta\right\rangle\right) \in\left[W_{\beta}\right]^{<\lambda}$. $\tau$ is a winning strategy if whenever P2 follows $\tau$, P2 wins.

$\mathcal{G}_{<\lambda}^{\eta}=\mathcal{G}_{<\lambda}^{\eta}(\infty)$ is the game played just as $\mathcal{G}_{<\lambda}^{\eta}(\kappa)$, except now P1 can choose partitions of any size. Let $\mathcal{G}_{\text {fin }}^{\eta}(\kappa)$ denote $\mathcal{G}_{<\omega}^{\eta}(\kappa)$. Jech's game $\mathcal{G}_{\text {fin }}(\kappa)$ in [4] is the same as $\mathcal{G}_{\text {fin }}^{\omega}(\kappa)$. Note that $\mathcal{G}_{<2}^{\eta}(\kappa)=\mathcal{G}_{1}^{\eta}(\kappa)$.

Remark. $\mathcal{G}_{<\lambda}^{\eta}$ can be played in a partial ordering in the natural way. As before, if $\mathbf{P}$ is separative, the existence of a winning strategy for $\mathrm{P} 1$ (P2) in $\mathcal{G}_{<\lambda}^{\eta}$ is invariant under the completion of $\mathbf{P}$. 
Note. In a $\max \left(\eta^{+}, \lambda\right)$-complete Boolean algebra, the following hold: For $\eta_{0} \leq$ $\eta_{1}, \kappa_{0} \leq \kappa_{1}$, and $\lambda_{0} \geq \lambda_{1}$, a winning strategy for P1 in $\mathcal{G}_{<\lambda_{0}}^{\eta_{0}}\left(\kappa_{0}\right)$ is a winning strategy for P1 in $\mathcal{G}_{<\lambda_{1}}^{\eta_{1}}\left(\kappa_{1}\right)$; conversely, a winning strategy for P2 in $\mathcal{G}_{<\lambda_{1}}^{\eta_{1}}\left(\kappa_{1}\right)$ is a winning strategy for P2 in $\mathcal{G}_{<\lambda_{0}}^{\eta_{0}}\left(\kappa_{0}\right)$. If $\mathrm{P} 2$ has a winning strategy in $\mathcal{G}_{<\lambda}^{\eta}(\kappa)\left(\mathcal{G}_{<\lambda}^{\eta}\right)$, then the $(\eta,<\lambda, \kappa)$-d.l. $((\eta,<\lambda, \infty)$-d.l. $)$ holds.

Theorems 1.3 and 1.4 (1) are consequences of the following two theorems. Theorem 2.2 is a generalization of a result of Jech [4 which states that the failure of the weak $(\omega, \kappa)$-d.l. implies the existence of a winning strategy for P1 in $\mathcal{G}_{\text {fin }}^{\omega}(\kappa)$.

Theorem 2.2. If $\mathbf{B}$ is $\max \left(\eta^{+}, \lambda\right)$-complete, $\lambda \leq \kappa$, and the $(\eta,<\lambda, \kappa)$-d.l. fails, then $P 1$ has a winning strategy in $\mathcal{G}_{<\lambda}^{\eta}(\kappa)$.

Proof. If the $(\eta,<\lambda, \kappa)$-d.l. fails, then there is a family $W_{\alpha}=\left\{b_{\alpha \beta}: \beta<\kappa\right\} \subseteq \mathbf{B}^{+}$, $(\alpha<\eta)$, such that $\bigvee_{f: \eta \rightarrow[\kappa]<\lambda} \bigwedge_{\alpha<\eta} \bigvee_{\beta \in f(\alpha)} b_{\alpha, \beta}=\mathbf{0}<\bigwedge_{\alpha<\eta} \bigvee_{\beta<\kappa} b_{\alpha, \beta}$. Let $a=\bigwedge_{\alpha<\eta} \bigvee_{\beta<\kappa} b_{\alpha \beta}$. P1 wins if P1 chooses $a$ at the beginning of the game, and $\forall \alpha<\eta, \mathrm{P} 1$ plays $W_{\alpha}$.

Theorem 2.3. If $\mathbf{B}$ is $\max \left(\eta^{+}, \lambda\right)$-complete, $\lambda \leq \kappa$, and P1 has a winning strategy in $\mathcal{G}_{<\lambda}^{\eta}(\kappa)$, then the $\left(\left(\kappa^{<\lambda}\right)^{<\eta},<\lambda, \kappa\right)$-d.l. fails in $\mathbf{B}$.

Proof. Suppose $\sigma$ is a winning strategy for P1. Let $\{a\}=\sigma(\mathbf{0})$ and $W_{\langle\rangle}=\sigma(\langle\rangle)$. Index the elements of $\left[W_{\langle\rangle}\right]^{<\lambda}$ using $s(0) \in \kappa^{<\lambda}$ so that $\left[W_{\langle\rangle}\right]^{<\lambda}=\left\{F_{\langle s(0)\rangle}: s(0) \in\right.$ $\left.\kappa^{<\lambda}\right\}$. Let $W_{\langle s(0)\rangle}=\sigma\left(\left\langle F_{\langle s(0)\rangle}\right\rangle\right)$ be the partition of $a$ which P1 chooses according to $\sigma$ if $\mathrm{P} 2$ has just chosen $F_{\langle s(0)\rangle} \in\left[W_{\langle\rangle}\right]<\lambda$. In general, for $\alpha<\eta$ and $s \in\left(\kappa^{<\lambda}\right)^{\alpha}$, given a sequence $\left\langle F_{s \uparrow 1}, F_{s \uparrow 2}, \ldots, F_{s \uparrow(\beta+1)}, \ldots: \beta<\alpha\right\rangle$ of P2's moves, let

$$
W_{s}=\sigma\left(\left\langle F_{s \uparrow(\beta+1)}: \beta<\alpha\right\rangle\right)
$$

be the partition of $a$ which P1 chooses according to $\sigma$. Index the elements of $\left[W_{s}\right]<\lambda$ using $s(\alpha) \in \kappa^{<\lambda}$ so that

$$
\left[W_{s}\right]^{<\lambda}=\left\{F_{s \frown s(\alpha)}: s(\alpha) \in \kappa^{<\lambda}\right\} .
$$

Note that $\left\{W_{s}: s \in\left(\kappa^{<\lambda}\right)^{<\eta}\right\}$ is a listing of all the possible choices for P1 under $\sigma$, and $\left\{F_{s}: s \in\left(\kappa^{<\lambda}\right)^{<\eta}\right.$ and $\operatorname{dom}(s)$ is a successor ordinal $\}$ is a listing of all the possible choices for P2 when P1 follows $\sigma$.

Claim. The $\left(\left(\kappa^{<\lambda}\right)^{<\eta},<\lambda, \kappa\right)$-d.l. fails for the partitions $W_{s}\left(s \in\left(\kappa^{<\lambda}\right)^{<\eta}\right)$ of $a$. For each $s \in\left(\kappa^{<\lambda}\right)^{<\eta}$, use $\kappa$ to index the elements of $W_{s}$ so that $W_{s}=\left\{b_{s, j}: j<\kappa\right\}$. It suffices to show that for every $f:\left(\kappa^{<\lambda}\right)^{<\eta} \rightarrow[\kappa]^{<\lambda}$,

$$
\bigwedge_{s \in(\kappa<\lambda)<\eta} \bigvee_{j \in f(s)} b_{s, j}=\mathbf{0} .
$$

Let $f:\left(\kappa^{<\lambda}\right)^{<\eta} \rightarrow[\kappa]^{<\lambda}$ be given. To show (21), we construct a sequence $t \in\left(\kappa^{<\lambda}\right)^{\eta}$ with the following two properties:

(a) $\forall \alpha<\eta, F_{t \uparrow(\alpha+1)}=\left\{b_{t\lceil\alpha, j}: j \in f(t\lceil\alpha)\}\right.$;

(b) $\langle a\rangle \frown\left\langle W_{t \uparrow \alpha}, F_{t \uparrow(\alpha+1)}: \alpha<\eta\right\rangle$ is a play of $\mathcal{G}_{<\lambda}^{\eta}(\kappa)$ in which P1 follows $\sigma$.

Let $t(0)$ be the unique element of $\kappa^{<\lambda}$ for which $F_{\langle t(0)\rangle}=\left\{b_{\langle\rangle, j}: j \in f(\langle\rangle)\right\}$. In general, for $\alpha<\eta$ and given $\langle t(\beta): \beta<\alpha\rangle$, let $t(\alpha)$ be the unique element of $\kappa^{<\lambda}$ for which

$$
F_{\langle t(\beta): \beta<\alpha\rangle-t(\alpha)}=\left\{b_{\langle t(\beta): \beta<\alpha\rangle, j}: j \in f(\langle t(\beta): \beta<\alpha\rangle)\right\} .
$$


Let

$$
t=\langle t(\alpha): \alpha<\eta\rangle \in\left(\kappa^{<\lambda}\right)^{\eta} .
$$

It follows from (19), (20), (22), and (23) that $t$ satisfies properties (a) and (b). Thus,

$$
\bigwedge_{s \in(\kappa<\lambda)<\eta} \bigvee_{j \in f(s)} b_{s, j} \leq \bigwedge_{\alpha<\eta} \bigvee F_{t \uparrow(\alpha+1)}=\mathbf{0},
$$

since property (a) implies the inequality and property (b) implies the equality in (24). Thus, (21) holds. Since $f$ was arbitrary, the $\left(\left(\kappa^{<\lambda}\right)<\eta,<\lambda, \kappa\right)$-d.l. fails for the partitions $W_{s}\left(s \in\left(\kappa^{<\lambda}\right)^{<\eta}\right)$ of $a$.

Corollary 2.4. If $\mathbf{B}$ is $\max \left(\eta^{+}, \lambda\right)$-complete and $\left(\kappa^{<\lambda}\right)^{<\eta}=\eta$, then the $(\eta,<\lambda, \kappa)$ d.l. holds in $\mathbf{B}$ if and only if $P 1$ does not have a winning strategy in $\mathcal{G}_{<\lambda}^{\eta}(\kappa)$.

Corollary 2.5 (GCH). Suppose $\mathbf{B}$ is $\max \left(\eta^{+}, \lambda\right)$-complete, $\lambda \leq \kappa$, and either (a) $\kappa^{+} \leq \eta$, or (b) $\kappa=\eta$ and $\eta$ is regular. Then the $(\eta,<\lambda, \kappa)-d . l$. holds in $\mathbf{B}$ if and only if $P 1$ does not have a winning strategy in $\mathcal{G}_{<\lambda}^{\eta}(\kappa)$.

It is not immediately clear whether the existence of a winning strategy for P2 is equivalent to the non-existence of a winning strategy for $\mathrm{P} 1$ in $\mathcal{G}_{<\lambda}^{\eta}(\kappa)$. For $\eta$ regular and $\lambda \leq \min (\kappa, \eta)$, I have constructed the following Suslin algebra in which they are not equivalent.

Example 2. If $\eta$ is regular, $\lambda \leq \min (\kappa, \eta)$, and $\nabla_{\eta^{+}}$holds, then there exists an $\eta^{+}$-Suslin algebra in which the game $\mathcal{G}_{<\lambda}^{\eta}(\kappa)$ is undetermined.

We construct a Suslin tree $(T, \preccurlyeq)=\left(\eta^{+}, \preccurlyeq\right)$ such that neither player has a winning strategy in $\mathcal{G}_{<\lambda}^{\eta}(\kappa)$ played in r.o. $\left(T^{*}\right)$, where $\left(T^{*}, \preccurlyeq^{*}\right)=(T, \succcurlyeq)$. In light of the natural correspondence between partitions of r.o. $\left(T^{*}\right)$ and partitions of levels of $T$, we need only work with partitions of levels of $T$. Since $T$ will satisfy the $\eta^{+}$-c.c. and P1 must choose partitions of levels of $T$ of cardinality $\leq \kappa$, we need only consider partitions of levels of $T$ of $\operatorname{size} \leq \mu=\min (\eta, \kappa)$. Every partition of some level of $T$ into $\leq \mu$-many pieces will be an element of the set $\left(\left[\eta^{+}\right] \leq \eta\right) \leq \mu$. $\nabla_{\eta^{+}}$ implies $\left|\left(\left[\eta^{+}\right] \leq \eta\right) \leq \mu\right|=\eta^{+}$, so we can index the elements of $\left(\left[\eta^{+}\right] \leq \eta\right) \leq \mu$ by

$$
\left(\left[\eta^{+}\right]^{\leq \eta}\right)^{\leq \mu}=\left\{W_{\alpha}: \alpha<\eta^{+}\right\}=\left\{\left\langle F_{\alpha, \beta}: \beta<\mu\right\rangle: \alpha<\eta^{+}\right\},
$$

where each $F_{\alpha, \beta} \in\left[\eta^{+}\right] \leq \eta$. It will be useful later that $\forall \alpha<\eta^{+}$, the elements of $W_{\alpha}$ are ordered.

The collection $\left\{W_{\alpha}: \alpha<\eta^{+}\right\}$will include all partitions of levels of $T$. The functions $f:\left(\eta^{+}\right)^{<\eta} \rightarrow \mu^{<\lambda}$ include all the strategies for P2. $\eta \geq \mu \geq \lambda$ and $\nabla_{\eta^{+}}$ imply $\left|\left(\eta^{+}\right)^{<\eta} \times \mu^{<\lambda}\right|=\eta^{+}$, so let $\phi:\left(\eta^{+}\right)^{<\eta} \times \mu^{<\lambda} \rightarrow \eta^{+}$be a bijection. For each $\alpha<\eta^{+}$, we will construct $\operatorname{Lev}(\alpha)=\eta \cdot(\alpha+1) \backslash \eta \cdot \alpha$ and a partition $\mathcal{P}_{\alpha}$ of $\operatorname{Lev}(\alpha)$. $\left\{\mathcal{P}_{\alpha}: \alpha<\eta^{+}\right\}$will be used later to show that P2 does not have a winning strategy.

Construction of $(T, \preccurlyeq)$ and $\left\{\mathcal{P}_{\alpha}: \alpha<\eta^{+}\right\}$: Let $\operatorname{Lev}(0)=\eta$. Let $\mathcal{P}_{0}=\left\langle P_{0, \beta}\right.$ : $\beta<\mu\rangle$ be a partition of $\operatorname{Lev}(0)$ into $\mu$-many non-empty subsets.

Let $\alpha<\eta$ and suppose $\operatorname{Lev}(\alpha)$ and $\mathcal{P}_{\alpha}$ have been constructed. Let $s: \eta \times \mu \rightarrow$ $\eta \cdot(\alpha+2) \backslash \eta \cdot(\alpha+1)$ be a bijection. $\forall \gamma<\eta$ let $S_{\gamma}=\{s(\gamma, \beta): \beta<\mu\}$. For each $\gamma<\eta$, let the immediate successors of $\eta \cdot \alpha+\gamma \in \operatorname{Lev}(\alpha)$ be the elements of $S_{\gamma}$. This constructs $\operatorname{Lev}(\alpha+1)$. For $\beta<\mu$, let $P_{\alpha+1, \beta}=\{s(\gamma, \beta): \gamma<\eta\}$, and let $\mathcal{P}_{\alpha+1}=\left\langle P_{\alpha+1, \beta}: \beta<\mu\right\rangle$. $\mathcal{P}_{\alpha+1}$ is a partition of $\operatorname{Lev}(\alpha+1)$ into $\mu$-many disjoint subsets, each of size $\eta$. 
Now consider a limit ordinal $\alpha<\eta^{+}$and suppose that $\forall \beta<\alpha, \operatorname{Lev}(\beta)$ and $\mathcal{P}_{\beta}$ have been constructed. Let $T_{\alpha}=\bigcup_{\beta<\alpha} \operatorname{Lev}(\beta)$. Let (a) be the statement, " $T_{\alpha}=\alpha,\left\{\mathcal{P}_{\beta}: \beta<\alpha\right\} \subseteq\left\{W_{\beta}: \beta<\alpha\right\}$, and $\phi^{\prime \prime}\left(\alpha^{<\eta} \times \mu^{<\lambda}\right)=\alpha$." If (a) does not hold, then for each $t \in T_{\alpha}$, pick one $\alpha$-branch $B_{t} \subseteq T_{\alpha}$ which contains $t$ and put one element of $\eta \cdot(\alpha+1) \backslash \eta \cdot \alpha$ on top of $B_{t}$ at level $\alpha$. Do this in such a way that $\operatorname{Lev}(\alpha)=\eta \cdot(\alpha+1) \backslash \eta \cdot \alpha$.

Suppose (a) holds. Let $\left\langle A_{\alpha}: \alpha<\eta^{+}\right\rangle$be a $\mho_{\eta^{+}}$-sequence fixed throughout the construction of $T$. Let (b) be the statement, " $A_{\alpha}$ is a maximal antichain in $T_{\alpha}$ ", and let (c) be the statement, " $A_{\alpha}=\phi(f) \cap \alpha$ for some $f:\left(\eta^{+}\right)^{<\eta} \rightarrow \mu^{<\lambda}$." Let $t \in T_{\alpha}$. If (b) holds, then $\exists u \in A_{\alpha}$ such that $t \preccurlyeq u$ or $t \succ u$; let $r \in T_{\alpha}$ such that $r \succcurlyeq t, u$. If (b) does not hold, then let $r=t$. If (c) does not hold, then pick $B_{t} \subseteq T_{\alpha}$ an $\alpha$-branch containing $r$ and put one element of $\eta \cdot(\alpha+1) \backslash \eta \cdot \alpha$ above $B_{t}$ at level $\alpha$. Otherwise, (c) holds. Let $\delta=\operatorname{ht}(r)$ and let $g: \delta+2 \rightarrow \eta^{+}$be the function such that $\forall \gamma \leq \delta+1, W_{g(\gamma)}=\mathcal{P}_{\gamma}$. If P1 plays the sequence $\left\langle\mathcal{P}_{\gamma}: \gamma \leq \delta+1\right\rangle$ and P2 follows $f$, then on the $\delta+1$-st round, $\mathrm{P} 2$ chooses

$$
\left\{F_{g(\delta+1), \beta}: \beta \in f(\langle g(\gamma): \gamma \leq \delta+1\rangle)\right\}=\left\{P_{\delta+1, \beta}: \beta \in f(\langle g(\gamma): \gamma \leq \delta+1\rangle)\right\} .
$$

Let $\zeta<\mu$ be such that $\zeta \notin f(\langle g(\gamma): \gamma \leq \delta+1\rangle)$. By construction, there exists exactly one successor of $r$ in $P_{\delta+1, \zeta}$, say $s$. Choose an $\alpha$-branch $B_{t} \subseteq T_{\alpha}$ which contains $s$ and put one element of $\eta \cdot(\alpha+1) \backslash \eta \cdot \alpha$ above $B_{t}$ at level $\alpha$.

Do this for each $t \in T_{\alpha}$ in such a manner that $\operatorname{Lev}(\alpha)=\eta \cdot(\alpha+1) \backslash \eta \cdot \alpha$. Now that $\operatorname{Lev}(\alpha)$ has been constructed, choose a partition $\mathcal{P}_{\alpha}=\left\langle P_{\alpha, \beta}: \beta<\mu\right\rangle$ of $\operatorname{Lev}(\alpha)$ into $\mu$-many non-empty, disjoint subsets.

Let $T=\bigcup_{\alpha<\eta^{+}} \operatorname{Lev}(\alpha)$. Let $C_{L}=\left\{\alpha<\eta^{+}: \alpha\right.$ is a limit ordinal $\}, C_{T}=$ $\left\{\alpha<\eta^{+}: T_{\alpha}=\alpha\right\}, C_{P}=\left\{\alpha<\eta^{+}:\left\{\mathcal{P}_{\gamma}: \gamma<\alpha\right\} \subseteq\left\{W_{\gamma}: \gamma<\alpha\right\}\right\}$, and $C_{\phi}=\left\{\alpha<\eta^{+}: \phi^{\prime \prime}\left(\alpha^{<\eta} \times \mu^{<\lambda}\right)=\alpha\right\}$. It is not hard to show that $C_{L}, C_{T}, C_{P}$, and $C_{\phi}$ are c.u.b. subsets of $\eta^{+}$. (Regularity of $\eta$ is necessary to ensure that $C_{\phi}$ is c.u.b.)

By the usual argument, $T$ is an $\eta^{+}$-Suslin tree. r.o. $\left(T^{*}\right)$ satisfies the $(\eta, \infty)$-d.l., so P1 does not have a winning strategy in $\mathcal{G}_{1}^{\eta}(\kappa)$, by Theorem $1.4(2)$. Since $\mathcal{G}_{1}^{\eta}(\kappa)$ is easier for P1 to win than $\mathcal{G}_{<\lambda}^{\eta}(\kappa), \mathrm{P} 1$ does not have a winning strategy in $\mathcal{G}_{<\lambda}^{\eta}(\kappa)$.

Claim. P2 does not have a winning strategy in $\mathcal{G}_{<\lambda}^{\eta}(\kappa)$ played in r.o. $\left(T^{*}\right)$. Let $f:\left(\eta^{+}\right)^{<\eta} \rightarrow \mu^{<\lambda}$ be a strategy for $\mathrm{P} 2$, and let

$$
\alpha \in C_{L} \cap C_{T} \cap C_{P} \cap C_{\phi} \cap\left\{\beta<\eta^{+}: \phi(f) \cap \beta=A_{\beta}\right\} .
$$

Let $p \in \operatorname{Lev}(\alpha), t \in T_{\alpha}$ such that $p$ is placed above $B_{t}$ in the construction of $\operatorname{Lev}(\alpha)$, $\delta=\operatorname{ht}(t)$, and $s \in B_{t} \cap \operatorname{Lev}(\delta+1)$. Let $g: \delta+2 \rightarrow \eta^{+}$be the function such that $\forall \beta \leq \delta+1, W_{g(\beta)}=\mathcal{P}_{\beta}$. Statements (a) and (c) hold for $\alpha$, so the construction of $\operatorname{Lev}(\alpha)$ ensures that $s \in P_{\delta+1, \zeta}$ for some $\zeta \notin f(\langle g(\gamma): \gamma \leq \delta+1\rangle)$. Thus, $s$ does not get chosen by $f$ when $\mathrm{P} 1$ plays the sequence $\left\langle\mathcal{P}_{\beta}: \beta<\alpha\right\rangle$; that is,

$$
s \notin \bigcup\left\{P_{\delta+1, \theta}: \theta \in f(\langle g(\gamma): \gamma \leq \delta+1\rangle)\right\} .
$$

$p \succ s$ implies there is no $\alpha$-branch in $\bigcap_{\beta<\alpha} \bigcup\left\{P_{\beta, \theta}: \theta \in f(\langle g(\gamma): \gamma \leq \beta\rangle)\right\}$ below $p$. Since this holds for all $p \in \operatorname{Lev}(\alpha), f$ is not a winning strategy for P2 in r.o. $\left(T^{*}\right)$. 


\section{OPEN PROBLEMS}

Example 2 reveals a curious fact: For $\eta$ regular, $(\eta, \infty)$-distributivity does not imply P2 has a winning strategy in the game $\mathcal{G}_{<\lambda}^{\eta}(\kappa)$, for any $\kappa$ and any $\omega<\lambda \leq$ $\min (\eta, \kappa)$, even though it is much easier for P2 to win $\mathcal{G}_{<\lambda}^{\eta}(\kappa)$ than $\mathcal{G}_{1}^{\eta}$.

Problem 1. Show within ZFC that the existence of a winning strategy for P2 in $\mathcal{G}_{<\lambda}^{\eta}(\kappa)$ is stronger than the $(\eta,<\lambda, \kappa)$-d.l.; or else find a model of ZFC in which they are equivalent.

Kamburelis showed that $\mathrm{P} 1$ does not have a winning strategy in $\mathcal{G}_{\text {fin }}^{\omega}(\kappa)$ iff the weak $(\omega, \kappa)$-d.l. holds and $V^{\mathbf{B}} \models[\kappa]^{\omega} \cap V$ is stationary [6].

Problem 2. Find a complete characterization of the non-existence of a winning strategy for $\mathrm{P} 1$ in $\mathcal{G}_{<\lambda}^{\eta}(\kappa)$ in terms of the $(\eta,<\lambda, \kappa)$-d.l. and an extra condition similar to that of Kamburelis.

\section{REFERENCES}

1. M. Foreman, Games played on Boolean algebras, J. Symbolic Logic 48 (3) (1983), 714-723. MR 85h:03064

2. S. Fuchino, H. Mildenberger, S. Shelah and P. Vojtáš, On absolutely divergent series, Fund. Math. 160 (3) (1999), 255-268. MR 2000g:03107

3. T. Jech, A game theoretic property of Boolean algebras, Logic Colloquium '77 (1978), 135-144. MR 80c:90184

4. (1984), 11-29. MR 85j:03110

5. , Distributive Laws, Handbook of Boolean Algebra, Vol. 2, North-Holland, Amsterdam, 1989, pp. 317-331. CMP 21:10

6. A. Kamburelis, On the weak distributivity game, Ann. Pure and Appl. Logic 66 (1994), 19-26. MR 95d:04004

7. S. Koppelberg, Handbook of Boolean Algebra, Vol. 1, North-Holland, Amsterdam, 1989. MR 90k:06002

Department of Mathematics, The Pennsylvania State University, 218 McAllister Building, University Park, Pennsylvania 16802

E-mail address: dobrinen@math.psu.edu 